\title{
A MOQFPP Method for Solving Quadratic Fractional Programming under Fuzzy Environment
}

\author{
Basiya K. Abdulrahim, Shorish O. Abdulla \\ Department of Mathematics, College of Education ,University of Garmian, Kurdistan Region -Iraq
}

\begin{abstract}
In this paper, a solution procedure is proposed to solve Fully Fuzzy Quadratic Fractional Programming problem (FFQFPP) where all the variables and parameters are triangular fuzzy numbers. Here, FFQFPP transformed into an equivalent Multi- Objective Quadratic Fractional Programming problem (MOQFPP). Then MOQFPP converted into an equivalent multi objective Quadratic programming problem by using Mathematical programming approach. The proposed solution illustrated through numerical examples.

Keywords: Quadratic programming problem, triangular fuzzy numbers, fuzzy mathematical programming.
\end{abstract}

\section{Introduction}

Nowadays, the problem of linear fractional programming has significant application in different real-life areas such as production planning, financial sector, health care, and all engineering field. In the practical applications, a model involves many para meters whose values are given by experts. However, both experts and decision makers frequently do not know the value of those parameters. To handle this type of situations, one may use the fuzzy nu mbers in the place of the crisp numbers. Thus, the crisp QFPP becomes a fuzzy Quadratic fractional programming problem (FQFPP) or fully fuzzy Quadratic fractional programming problem (FFQFPP). Hence, FFQFP is an interesting research area in the recent years. In (2017) Das and Manda a MOLFP Method for Solving Linear Fractional Programming under Fuzzy Environment [2]. In (1992) Dutta, Tiwari, and Rao MOLFPP a fuzzy set theoretic approach [3]. In (2008) Fukushima and Hayashi Quadratic Fractional Programming Problems with Quadratic Constraints [4]. In (2014) Lachhwani FGP Approach to Multi Objective Quadratic Fractional Programming Problem [5]. In (2017) Osman, Emam and El Sayed Multi-level Multi-objective QFPP with Fuzzy Parameters: A FGP Approach [6]. In (2014) Rashidul Hasan and Babul Hasan An Alternative Method for Solving Quadratic Fractional Programming Problems with Homogenous Constraints [7]. In (2003) Stancu-Minasian and Pop On a fuzzy set approach to solving multiple objective linear fractional programming problem [8]. In (2014) Veeramani and Sumathi Fuzzy mathematical programming approach for solving fuzzy linear fractional programming problem [9]. In this paper, we consider the fully fuzzy Quadratic fractional programming problem (FFQFPP). In this paper, we consider the fully fuzzy Quadratic fractional progra mming problem. First, the FFQFPP transform into a fuzzy linear fractional programming problem (FLFPP) with three crisp objective functions. Then the FQFPP will be converted into a multi-objective Quadratic fractional programming problem (MOQFPP). We also prove that this solution can be considered as an exact solution of FFQFPP. Finally, we show the advantages of the proposed method, numerical example is solved. This paper is organized as follows, some basic definitions and notations are present in Section 2. In Section 3, we discuss the QFPP. In Section 4, we present our proposed method. A real-life example is provided to validate the proposed method in Section 5. Finally, the conclusion is given in Section 6. 


\section{Preliminaries}

We have presented some basics concept of fuzzy triangular number, which was very useful in this paper [1].

Definition 2.1: Let $X$ denotes a universal set. Then a fuzzy subset $\tilde{A}$ of $X$ is defined by its membership function $u_{\tilde{A}}: X \rightarrow[0,1]$, which assigned a real number $u_{\tilde{A}}(X)$ in the interval $[0,1]$, to each element $x \in X$, where the values of $u_{\tilde{A}}(X)$ at $x$ shows the grade of membership of $x$ in $\tilde{A}$. A fuzzy subset $\tilde{A}$ can be characterized as a set of ordered pairs of elements $x$ and grade $u_{\widetilde{A}}(X)$ and is often written $\widetilde{A}=$ $\left(x, u_{\tilde{A}}(X)\right): x \in X$ is called a fuzzy set.

Definition 2.2: A fuzzy number $\tilde{A}=(b, c, a)$ is said to be a triangular fuzzy number if its membership function is given by:

$u_{\tilde{A}}(X)= \begin{cases}\frac{(x-b)}{(c-b)} & b \leq x \leq c \\ \frac{(x-a)}{(c-b)} & c \leq x \leq a \\ 0 & \text { else }\end{cases}$

Definition 2.3: Two triangular fuzzy number $\tilde{A}=(b, c, a)$ and $\widetilde{B}=(e, f, d)$ are said to be equal if and only if $b=d, c=f, a=d$.

Definition 2.4: Let $\tilde{A}=(b, c, a)$ and $\widetilde{B}=(e, f, d)$ be two triangular fuzzy numbers then:

$\tilde{A}+\tilde{B}=(b, c, a)+(e, f, d)=(b+e, c+f, a+d)$

$\tilde{A}-\tilde{B}=(b, c, a)-(e, f, d)=(b-e, c-f, a-d)$

If $\tilde{A}=(b, c, a)$ be any triangular fuzzy number and $\tilde{B}=(e, f, d)$ be a non-negative triangular fuzzy number, then:

$\tilde{A} \otimes \tilde{B}=\tilde{A} \tilde{B}= \begin{cases}(b e, c f, a d) & \text { if } b \geq 0 \\ (b d, c f, a d) & \text { if } b<0, a \geq 0 \\ (b d, c f, c d) & \text { if } c<0\end{cases}$

Definition 2.5: Let $\tilde{A}=(b, c, a)$ and $\tilde{B}=(e, f, d)$ be two triangular fuzzy numbers. We say that $\tilde{A}$ is relatively less than $\widetilde{B}$, if and only if:

$$
\begin{array}{ll}
\text { i. } & b<e \text { or } \\
\text { ii. } & b=e \text { and }(b-c)>(e-f) \text { or } \\
\text { i. } & b=e \text { and }(b-c)=(e-f), \text { and }(a+d)=(d+e)
\end{array}
$$

Note: It is clear from the definition 2.7 that $b=e$ and $(b-c)=(e-f)$, and $(a+d)=(d+e)$ if and only if $\tilde{A}=\tilde{B}$.

\section{Quadratic Fractional Programming Problem}

\section{Definition 3.1: Quadratic Programming Problem}

If the optimization problem is of the form

Maximize (Minimize) $Z=\alpha+C^{T} x+\frac{1}{2} x^{T} G x$

Subject to :

$$
A x\left[\begin{array}{l}
\leq \\
\geq \\
=
\end{array}\right] b
$$

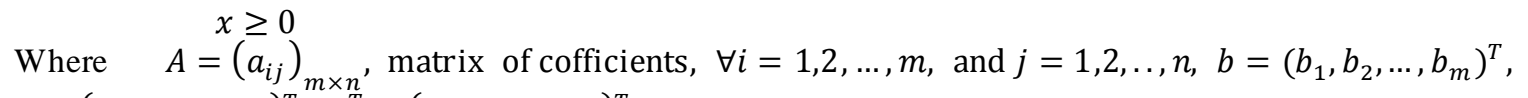
$x=\left(x_{1}, x_{2}, \ldots, x_{n}\right)^{T}, C^{T}=\left(c_{1}, c_{2}, \ldots, c_{n}\right)^{T}$, and $G=\left(g_{i i}\right)_{n \times n}$ is a positive definite or positive semidefinite symmetric square matrix, and $T$ is transposed then the constraints are linear and the objective function is quadratic. Such an optimization problem is said to be a QPP [1].

Definition 3.2: Quadratic Fractional Programming Problem The mathematical programming problem for QFPP can be formulated as follows 
Maximize (Minimize) $Z=\frac{\alpha_{1}+C_{1}^{T} x+\frac{1}{2} x^{T} G_{1} x}{\alpha_{2}+C_{2}^{T} x+\frac{1}{2} x^{T} G_{2} x}$

Subject to :

$$
\begin{gathered}
A x\left[\begin{array}{l}
\leq \\
\geq \\
=
\end{array}\right] b \\
x \geq 0
\end{gathered}
$$

Where $G_{1}, G_{2}$ are $(n \times n)$ matrix of coefficients with $G_{1}, G_{2}$ are symmetric matrixes. All vectors are assumed to be column vectors unless transposed $(T)$, where $x$ is an $n$-dimensional vector of decision variables, $C_{1}, C_{2}$ is the $n$-dimensional vector of constants, $b$ is $m$-dimensional vector of constants, $\alpha_{1}, \alpha_{2}$ are scalars [1]. In this work the problem that has objective function is tried to be solved has the following form:

Maximize $Z=\frac{\left(c^{T} x+\gamma\right)\left(e^{T} x+\delta\right)}{\left(d^{T} x+\beta\right)\left(f^{T} x+\varepsilon\right)}=\left(\frac{\left(c^{T} x+\gamma\right)}{\left(d^{T} x+\beta\right)}\right) *\left(\frac{\left(e^{T} x+\delta\right)}{\left(f^{T} x+\varepsilon\right)}\right)=Z_{1} \times Z_{2}$

or Maximize $Z=\frac{\left(c^{T} x+\gamma\right)\left(e^{T} x+\delta\right)}{\left(d^{T} x+\beta\right)\left(f^{T} x+\varepsilon\right)}=\left(\frac{\left(c^{T} x+\gamma\right)}{\left(f^{T} x+\varepsilon\right)}\right) *\left(\frac{\left(e^{T} x+\delta\right)}{\left(d^{T} x+\beta\right)}\right)=Z_{1} \times Z_{2}$

Subject to:

$$
\begin{gathered}
A x\left[\begin{array}{l}
\leq \\
\geq \\
=
\end{array}\right] b \\
x \geq 0
\end{gathered}
$$

Where $x \in R^{n}, A$ is an $m \times n$ matrix; $c, e, d$ and $f$ are $n$-vectors; $b \in R^{m}$ and $\gamma, \beta, \delta$, $\varepsilon$ are scalar constants. Moreover $f^{T} x+\varepsilon, d^{T} x+\beta>0$ everywhere in $x$.

Now, in order to solve the above QFPP by LFPP technique, we decompose it into two Linear fractional objectives of LFP problems namely $Z_{1}$ and $Z_{2}$ as given below:

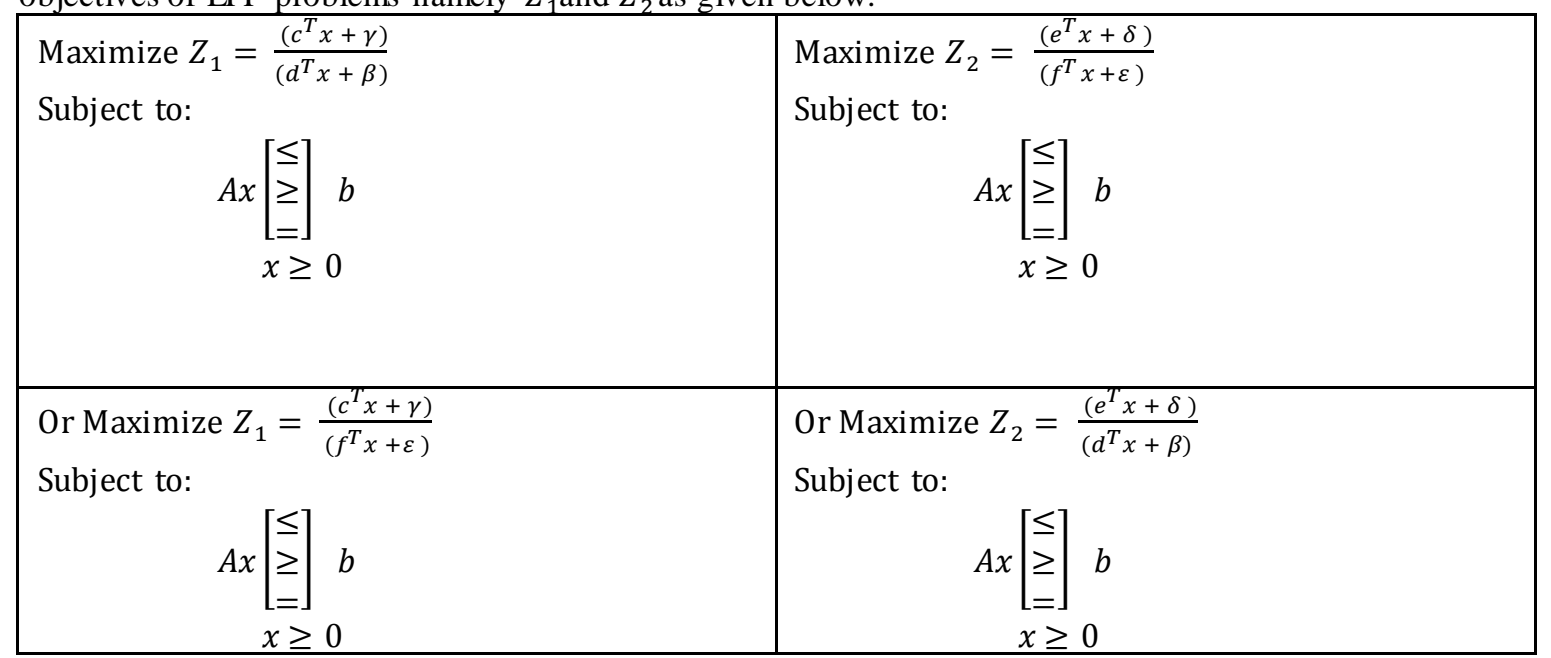

The mathematical programming problem for QFPP can be formulated two parts LFPP as follows respectively:

Maximize $Z_{1}=\frac{\left(c^{T} z+\gamma\right)}{\left(d^{T} z+\beta\right)}=\frac{F(z)}{G(z)}$ and Maximize $Z_{2}=\frac{e^{T} z+\delta}{f^{T} z+\varepsilon}$

Subject to:

$$
A z\left[\begin{array}{l}
\leq \\
\geq \\
=
\end{array}\right] b
$$

Where, $z, c^{T}, d^{T} \underset{\geq}{\geq} \in R^{n}, A \in R^{n+m}, \gamma, \beta \in R$. 
For some values of $z, G(z)$ may be equal to zero. To avoid such cases, one requires that either: $\{z \geq$ $\left.0, A z\left[\begin{array}{l}\leq \\ \geq \\ =\end{array}\right] b, G(z)>0\right\}$ or $\left\{z \geq 0, A z\left[\begin{array}{l}\leq \\ \geq \\ =\end{array}\right] b, G(z)<0\right\}$

For satisfaction, we assume that (2.1) satisfies the condition that:

$$
\left\{z \geq 0, A z\left[\begin{array}{l}
\leq \\
\geq \\
=
\end{array}\right] b, G(z)>0\right\}
$$

Theorem 3.1 [1]: Assume that no point $(x, 0)$ with $x \geq 0$ is feasible for the following linear programming problem (LPP):

Maximize $Z_{1}=c^{T} x+\gamma t$

Subject to:

$$
\begin{aligned}
& d^{T} z+\beta t=1 \\
& A x-b t=0 \\
& t \geq 0, x \geq 0
\end{aligned}
$$

Now assume that the equation (3.6) then the LFPP (2.1) us equivalent into linear programming problem (3.7).

\section{Fully Fuzzy Quadratic Fractional Programming Problem}

Quadratic fractional programming problem is evidently an uncertain optimization problem due to its variations in the maximum daily requirements. So, the amount of each product of ingredient will be uncertain. Hence, we will model the fully fuzzy Quadratic fractional programming problem where all the variables and all the parameters are triangular fuzzy numbers to avoid uncertain. Let us consider a general format of fully fuzzy Quadratic fractional programming problem can be formulated two parts LFPP as follows respectively:

Maximize $Z_{1}=\frac{\left(\tilde{c}^{T} \tilde{x}+\tilde{\gamma}\right)}{\left(\tilde{d}^{T} \tilde{x}+\widetilde{\beta}\right)}$ and Maximize $Z_{2}=\frac{\left(\tilde{e}^{T} \tilde{x}+\widetilde{\delta}\right)}{\left(\tilde{f}^{T} \tilde{x}+\tilde{\varepsilon}\right)}$

Subject to:

$$
\begin{aligned}
\tilde{A} \tilde{x}\left[\begin{array}{l}
\leq \\
\geq \\
=
\end{array}\right] \quad \tilde{b} \\
\tilde{x} \geq 0
\end{aligned}
$$

Consider the equation (2.4) and let $\tilde{x}=\left(x^{*}, y^{*}, z^{*}\right)$ be an optimal solution of this FFLFP. Furthermore, let all the parameters $\tilde{x}, \tilde{c}, \tilde{q}, \tilde{d}, \tilde{r}, \tilde{b}$ and $\tilde{Z}=Z_{1} \times Z_{2}$ are represented by triangular fuzzy numbers

$(x, y, z),(p, q, r),\left(\alpha_{1}, \alpha_{2}, \alpha_{3}\right),\left(\beta_{1}, \beta_{2}, \beta_{3}\right),\left(b_{1}, b_{2}, b_{3}\right),(u, v, w)$ and $\left(z_{1}, z_{2}, z_{3}\right)$ respectively. Then we can rewrite the mentioned FFLFP as follows:

Maximize $\left(z_{1}, z_{2}, z_{3}\right)=\frac{(p, q, r)^{T} \otimes(x, y, z)+\left(\alpha_{1}, \alpha_{2}, \alpha_{3}\right)}{(u, v, w)^{T} \otimes(x, y, z)+\left(\beta_{1}, \beta_{2}, \beta_{3}\right)}$

Subject to:

$$
\begin{gathered}
(b, c, a) \otimes(x, y, z)\left[\begin{array}{l}
\leq \\
\geq \\
=
\end{array}\right]\left(b_{1}, b_{2}, b_{3}\right) \\
(x, y, z) \geq 0
\end{gathered}
$$

The proposed approach for solving FFQFPP can be formulated two parts FFLFPP consists of the following steps summarized as follows:

Step 1: The above problem is converted into MOLFP problem as follows:

Maximize $\left(z_{1}, z_{2}, z_{3}\right)=\left\{\frac{p^{T} x+\alpha_{1}}{u^{T} x+\beta_{1}}, \frac{q^{T} y+\alpha_{2}}{v^{T} y+\beta_{2}}, \frac{r^{T} z+\alpha_{3}}{w^{T} z+\beta_{3}}\right\}$

Subject to:

$$
\begin{aligned}
& b \otimes x \leq b_{1} \\
& c \otimes y \leq b_{2} \\
& a \otimes z \leq b_{3} \\
& (x, y, z) \geq 0
\end{aligned}
$$


Step 2: With respect to definition 2.2 and 2.3 the problem in Step 1, can be rewritten as:

Maximize $\left(z_{1}, z_{2}, z_{3}\right)=\left\{\left(p^{T} x+\alpha_{1}\right), q^{T} y+\alpha_{2},\left(r^{T} z+\alpha_{3}\right)\right\}$

Subject to:

$$
\begin{aligned}
& b \otimes x-b_{1} \leq 0 \\
& c \otimes y-b_{2} \leq 0 \\
& a \otimes z-b_{3} \leq 0 \\
& \left(u^{T} x+\beta_{1}\right) \leq 1 \\
& \left(v^{T} y+\beta_{2}\right) \leq 1 \\
& \left(w^{T} z+\beta_{3}\right) \leq 1 \\
& (x, y, z) \geq 0
\end{aligned}
$$

Step 3: Regarding the definition 2.6 the problem in Step 2 is converted to the MOLPP with three crisp objective functions and the constraints are changed as follows:

$$
\operatorname{Maximize}\left(z_{1}, z_{2}, z_{3}\right)=\left\{\begin{array}{l}
\left(p^{T} x+\alpha_{1}\right) \\
\left(p^{T} x+\alpha_{1}\right)-\left(q^{T} y+\alpha_{2}\right) \\
\left(q^{T} y+\alpha_{2}\right)+\left(r^{T} z+\alpha_{3}\right)
\end{array}\right\}
$$

Subject to:

$$
\begin{aligned}
& b \otimes x-b_{1} \leq 0 \\
& \left(b \otimes x-b_{1}\right)-\left(c \otimes y-b_{2}\right) \leq 0 \\
& \left(b \otimes x-b_{1}\right)+\left(a \otimes z-b_{3}\right) \leq 0 \\
& \left(u^{T} x+\beta_{1} t\right) \leq 1 \\
& \left(u^{T} x+\beta_{1} t\right)-\left(v^{T} y+\beta_{2} t\right) \leq 0 \\
& \left(u^{T} x+\beta_{1} t\right)+\left(w^{T} z+\beta_{3} t\right) \leq 2 \\
& (x, y, z) \geq 0
\end{aligned}
$$

Step 4: Solving the problem in Step 3 by MATLAB software, we get the solution.

\section{Numerical Examples}

Example 5.1: We consider the following QFPP as In Jamshedpur City, India, A Wooden company is the producer of two kinds of products $A$ and $B$ with profit around $[(5,1,3),(5,1,3)]$ and around $[(4,1,6),(4,1,6)]$ dollar per unit, respectively. However, the cost for each one unit of the above products is around $[(4,6,5),(4,6,5)]$ and around $[(6,3,9),(6,3,9)]$ dollars respectively. It is assuming that a fixed cost of around $[(1,2,6),(1,2,6)]$ dollar is added to the cost function due to expected duration through the process of production. Suppose the raw material needed for manufacturing product A and B is about $(3,2,1)$ units per pound and about $(6,4,1)$ units per dollar respectively, the supply for th is raw material is restricted to about $(13,5,2)$ dollar. Man-hours per unit for the product $\mathrm{A}$ is about $(4,1,2)$ hour and product $\mathrm{B}$ is about $(6,5,4)$ hour per unit for manufacturing but total Man hour available is about $(6,3,9)$ hour daily. Determine how many products A and B should be manufactured in order to maximize the total profit.

Solution 5.1: Solving the example 5.1 by using feasible direction method as follows. This real-life problem can be formulated to the following QFPP:

$$
\text { Maximize } Z=\frac{(5,1,3)\left(x_{1}, y_{1}, z_{1}\right)+(4,1,6)\left(x_{2}, y_{2}, z_{2}\right)}{(4,6,5)\left(x_{1}, y_{1}, z_{1}\right)+(6,3,9)\left(x_{2}, y_{2}, z_{2}\right)+(1,2,6)} \times \frac{(5,1,3)\left(x_{1}, y_{1}, z_{1}\right)+(4,1,6)\left(x_{2}, y_{2}, z_{2}\right)}{(4,6,5)\left(x_{1}, y_{1}, z_{1}\right)+(6,3,9)\left(x_{2}, y_{2}, z_{2}\right)+(1,2,6)}
$$

Subject to:

$$
\begin{aligned}
& (3,2,1)\left(x_{1}, y_{1}, z_{1}\right)+(6,4,1)\left(x_{2}, y_{2}, z_{2}\right) \leq(13,5,2) \\
& (4,1,2)\left(x_{1}, y_{1}, z_{1}\right)+(6,5,4)\left(x_{2}, y_{2}, z_{2}\right) \leq(6,3,9) \\
& \left(x_{1}, y_{1}, z_{1}\right),\left(x_{2}, y_{2}, z_{2}\right) \geq 0
\end{aligned}
$$

The problem (5.13) is converted into the MOQFPP as follows: 
Maximize $Z=\left\{\frac{5 x_{1}+4 x_{2}}{4 x_{1}+6 x_{2}+1} \times \frac{5 x_{1}+4 x_{2}}{4 x_{1}+6 x_{2}+1}, \frac{y_{1}+y_{2}}{6 y_{1}+3 y_{2}+2} \times \frac{y_{1}+y_{2}}{6 y_{1}+3 y_{2}+2}, \frac{3 z_{1}+6 z_{2}}{5 z_{1}+9 z_{2}+6} \times \frac{3 z_{1}+6 z_{2}}{5 z_{1}+9 z_{2}+6}\right\}$

Subject to:

We can write

$$
\begin{aligned}
& 3 x_{1}+6 x_{2} \leq 13 \\
& 2 y_{1}+4 y_{2} \leq 5 \\
& z_{1}+z_{2} \leq 2 \\
& 4 x_{1}+6 x_{2} \leq 6 \\
& y_{1}+5 y_{2} \leq 3 \\
& 2 z_{1}+4 z_{2} \leq 9 \\
& \left(x_{1}, y_{1}, z_{1}\right),\left(x_{2}, y_{2}, z_{2}\right) \geq 0
\end{aligned}
$$

Maximize $\mathrm{Z}_{1}=\frac{(5,1,3)\left(x_{1}, y_{1}, z_{1}\right)+(4,1,6)\left(x_{2}, y_{2}, z_{2}\right)}{(4,6,5)\left(x_{1}, y_{1}, z_{1}\right)+(6,3,9)\left(x_{2}, y_{2}, z_{2}\right)+(1,2,6)}$

Subject to:

$$
\begin{aligned}
& (3,2,1)\left(x_{1}, y_{1}, z_{1}\right)+(6,4,1)\left(x_{2}, y_{2}, z_{2}\right) \leq(13,5,2) \\
& (4,1,2)\left(x_{1}, y_{1}, z_{1}\right)+(6,5,4)\left(x_{2}, y_{2}, z_{2}\right) \leq(6,3,9) \\
& \left(x_{1}, y_{1}, z_{1}\right),\left(x_{2}, y_{2}, z_{2}\right) \geq 0
\end{aligned}
$$

Maximize $\mathrm{Z}_{2}=\frac{(5,1,3)\left(x_{1}, y_{1}, z_{1}\right)+(4,1,6)\left(x_{2}, y_{2}, z_{2}\right)}{(4,6,5)\left(x_{1}, y_{1}, z_{1}\right)+(6,3,9)\left(x_{2}, y_{2}, z_{2}\right)+(1,2,6)}$

Subject to:

$$
\begin{aligned}
& (3,2,1)\left(x_{1}, y_{1}, z_{1}\right)+(6,4,1)\left(x_{2}, y_{2}, z_{2}\right) \leq(13,5,2) \\
& (4,1,2)\left(x_{1}, y_{1}, z_{1}\right)+(6,5,4)\left(x_{2}, y_{2}, z_{2}\right) \leq(6,3,9) \\
& \left(x_{1}, y_{1}, z_{1}\right),\left(x_{2}, y_{2}, z_{2}\right) \geq 0
\end{aligned}
$$

The First problem (5.14a) is converted into the MOLFPP as follows:

Maximize $Z_{1}=\left\{\frac{5 x_{1}+4 x_{2}}{4 x_{1}+6 x_{2}+1}, \frac{y_{1}+y_{2}}{6 y_{1}+3 y_{2}+2}, \frac{3 z_{1}+6 z_{2}}{5 z_{1}+9 z_{2}+6}\right\}$

Subject to:

$$
\begin{aligned}
& 3 x_{1}+6 x_{2} \leq 13 \\
& 2 y_{1}+4 y_{2} \leq 5 \\
& z_{1}+z_{2} \leq 2 \\
& 4 x_{1}+6 x_{2} \leq 6 \\
& y_{1}+5 y_{2} \leq 3 \\
& 2 z_{1}+4 z_{2} \leq 9 \\
& \left(x_{1}, y_{1}, z_{1}\right),\left(x_{2}, y_{2}, z_{2}\right) \geq 0
\end{aligned}
$$

The problem (5.15) is transformed into an equivalent MOLPP as follows:

$$
\text { Max. } z_{1}=5 y_{1}+4 y_{2}
$$

Maximize $Z_{1}=\operatorname{Max} \cdot z_{2}=z+z_{2}$

Subject to:

$$
\operatorname{Max} . z_{3}=3 x_{1}+6 x_{2}
$$

$$
\begin{aligned}
& 4 y_{1}+6 y_{2}+t \leq 1 \\
& 6 z_{1}+3 z_{2}+2 t \leq 1 \\
& 5 x_{1}+9 x_{2}+6 t \leq 1 \\
& 3 y_{1}+6 y_{2}-13 t \leq 0 \\
& 2 z_{1}+4 z_{2}-5 t \leq 0 \\
& x_{1}+x_{2}-2 t \leq 0 \\
& 4 y_{1}+6 y_{2}-6 t \leq 0 \\
& z_{1}+5 z_{2}-3 t \leq 0 \\
& 2 x_{1}+4 x_{2}-9 t \leq 0 \\
& \left(x_{1}, y_{1}, z_{1}\right),\left(x_{2}, y_{2}, z_{2}\right) \geq 0
\end{aligned}
$$

The problem (5.16) can be written as follows: 


$$
\text { Max. } z_{1}=5 y_{1}+4 y_{2}
$$

Maximize $z_{1}=$ Max. $z_{2}=5 y_{1}+4 y_{2}-z_{1}-z_{2}$

$$
\text { Max. } z_{3}=5 y_{1}+4 y_{2}+3 x_{1}+6 x_{2}
$$

Subject to:

$$
\begin{aligned}
& 4 y_{1}+6 y_{2}+t \leq 1 \\
& 4 y_{1}+6 y_{2}+t-6 z_{1}-3 z_{2}-2 t \leq 0 \\
& 4 y_{1}+6 y_{2}+t+5 x_{1}+9 x_{2}+6 t \leq 2 \\
& 3 y_{1}+6 y_{2}+13 t \leq 0 \\
& 3 y_{1}+6 y_{2}+13 t-2 z_{1}-4 z_{2}+5 t \leq 0 \\
& 3 y_{1}+6 y_{2}-13 t+x_{1}+x_{2}-2 t \leq 0 \\
& 4 y_{1}+6 y_{2}-6 t \leq 0 \\
& 4 y_{1}+6 y_{2}-6 t-z_{1}-5 z_{2}+3 t \leq 0 \\
& 4 y_{1}+6 y_{2}-6 t+2 x_{1}+4 x_{2}-9 t \leq 0 \\
& \left(x_{1}, y_{1}, z_{1}\right),\left(x_{2}, y_{2}, z_{2}\right) \geq 0
\end{aligned}
$$

Solving the problem (5.17) we get $y_{1}=0.214, y_{2}=0, x_{1}=0, x_{2}=0.016, z_{1}=0.067, z_{2}=0.102, z_{3}=$ $0,09, t=0.142$. The Second problem $(5.14 \mathrm{~b})$ is converted into the MOLFPP after similarly second part solve we get $y_{1}=0.214, y_{2}=0, x_{1}=0, x_{2}=0.016, z_{1}=0.067, z_{2}=0.102, z_{3}=0.09, t=0.142$. Hence the solution of the problem (13) is $z_{1}=1.1449, z_{2}=0.028561, z_{3}=0.0081$.

Example 5.2: We consider the following QFPP as. Let us consider the following Quadratic fractional programming as follows:

Maximize $Z=\frac{(0,1,2) \tilde{x}_{1}-(-2,-1,0) \tilde{x}_{2}+(0,1,2)}{(0,1,2) \tilde{x}_{1}+(0,1,2) \tilde{x}_{2}+(1,2,3)} \times \frac{(0,1,2) \tilde{x}_{1}-(-2,-1,0) \tilde{x}_{2}+(0,1,2)}{(0,1,2) \tilde{x}_{1}+(0,1,2) \tilde{x}_{2}+(1,2,3)}$

Subject to:

$$
\begin{aligned}
& (1,2,3) \tilde{x}_{1}+(1,2,3) \tilde{x}_{2} \leq(1,2,3) \\
& (0,1,2) \tilde{x}_{1}-(-2,-1,0) \tilde{x}_{2} \leq(0,1,2) \\
& \tilde{x}_{1}, \tilde{x}_{2} \geq 0
\end{aligned}
$$

Solution 5.2: Solving

the example 5.2 by using feasible direction method as follows. We can write Maximize $\mathrm{Z}_{1}=\frac{(0,1,2) \tilde{x}_{1}-(-2,-1,0) \tilde{x}_{2}+(0,1,2)}{(0,1,2) \tilde{x}_{1}+(0,1,2) \tilde{x}_{2}+(1,2,3)}$

Subject to:

$$
\begin{aligned}
& (1,2,3) \tilde{x}_{1}+(1,2,3) \tilde{x}_{2} \leq(1,2,3) \\
& (0,1,2) \tilde{x}_{1}-(-2,-1,0) \tilde{x}_{2} \leq(0,1,2) \\
& \tilde{x}_{1}, \tilde{x}_{2} \geq 0
\end{aligned}
$$

Maximize $Z_{2}=\frac{(0,1,2) \tilde{x}_{1}-(-2,-1,0) \tilde{x}_{2}+(0,1,2)}{(0,1,2) \tilde{x}_{1}+(0,1,2) \tilde{x}_{2}+(1,2,3)}$

Subject to:

$$
\begin{aligned}
& (1,2,3) \tilde{x}_{1}+(1,2,3) \tilde{x}_{2} \leq(1,2,3) \\
& (0,1,2) \tilde{x}_{1}-(-2,-1,0) \tilde{x}_{2} \leq(0,1,2) \\
& \tilde{x}_{1}, \tilde{x}_{2} \geq 0
\end{aligned}
$$

Now the problem (5.19a) is as follows

Maximize $\mathrm{Z}_{1}=\frac{(0,1,2) \otimes\left(\left(x_{1}\right)^{p},\left(x_{1}\right)^{q},\left(x_{1}\right)^{r}\right)-(-2,-1,0) \otimes\left(\left(x_{2}\right)^{p},\left(x_{2}\right)^{q},\left(x_{2}\right)^{r}\right)+(0,1,2)}{(0,1,2) \otimes\left(\left(x_{1}\right)^{p},\left(x_{1}\right)^{q},\left(x_{1}\right)^{r}\right)+(0,1,2) \otimes\left(\left(x_{2}\right)^{p},\left(x_{2}\right){ }^{q},\left(x_{2}\right)^{r}\right)+(1,2,3)}$

Subject to:

$$
\begin{aligned}
& (1,2,3) \otimes\left(\left(x_{1}\right)^{p},\left(x_{1}\right)^{q},\left(x_{1}\right)^{r}\right)+(1,2,3) \otimes\left(\left(x_{2}\right)^{p},\left(x_{2}\right)^{q},\left(x_{2}\right)^{r}\right) \leq(1,2,3) \\
& (0,1,2) \otimes\left(\left(x_{1}\right)^{p},\left(x_{1}\right)^{q},\left(x_{1}\right)^{r}\right)-(-2,-1,0) \otimes\left(\left(x_{2}\right)^{p},\left(x_{2}\right)^{q},\left(x_{2}\right)^{r}\right) \leq(0,1,2) \\
& \tilde{x}_{1}, \tilde{x}_{2} \geq 0
\end{aligned}
$$

According to Step 2 and Step 3, we get the following MOLPP: 


$$
\begin{aligned}
\text { Max. } z_{1} & =-2\left(y_{2}\right)^{p} \\
\text { Maximize } \mathrm{Z}_{1}= & \text { Max. } z_{2}=\left(y_{1}\right)^{q}-\left(y_{2}\right)^{q}+t_{2} \\
& \text { Max. } z_{3}=2\left(y_{1}\right)^{r}+2 t_{3}
\end{aligned}
$$

Subject to:

$$
\begin{aligned}
& 3 t_{3} \leq 0 \\
& \left(y_{1}\right)^{q}+\left(y_{2}\right)^{q}-2 t_{2} \leq 0 \\
& 2\left(y_{1}\right)^{r}+2\left(y_{2}\right)^{r}-t_{1} \leq 0 \\
& -2\left(y_{1}\right)^{r}-2 t_{3} \leq 0 \\
& \left(y_{1}\right)^{q}+\left(y_{2}\right)^{q}-t_{2} \leq 0 \\
& -2\left(y_{1}\right)^{r} \leq 0 \\
& t_{1} \leq 1 \\
& \left(y_{1}\right)^{q}+\left(y_{2}\right)^{q}+2 t_{2} \leq 1 \\
& \left(y_{1}\right)^{r}+\left(y_{2}\right)^{r}+3 t_{3} \leq 1 \\
& (y)^{q}-(y)^{p} \geq 0,(y)^{r}-(y)^{q} \geq 0,(y)^{p} \geq 0 t_{1}, t_{2}, t_{3} \geq 0
\end{aligned}
$$

Using Steps 3, Step 4, Step 5 and Step 6, the optimal solution of the problem is:

$$
\begin{aligned}
& \tilde{y}^{*}=\left\{\check{y}_{1}^{*}=\left(y_{1}^{*}\right)^{p},\left(y_{1}^{*}\right)^{q},\left(y_{1}^{*}\right)^{r}\right\}=(0,0.2,0.2) \\
& \tilde{y}^{*}=\left\{\tilde{y}_{2}^{*}=\left(y_{2}^{*}\right)^{p},\left(y_{2}^{*}\right)^{q},\left(y_{2}^{*}\right)^{r}\right\}=(0,0,0)
\end{aligned}
$$

$t^{*}=\left(t_{1}, t_{2}, t_{3}\right)=(0.1,0.2,0.2)$

Then the solution of the problem

$$
\begin{aligned}
& \tilde{x}^{*}=\left\{\tilde{x}_{1}^{*}=\left(x_{1}^{*}\right)^{p},\left(x_{1}^{*}\right)^{q},\left(x_{1}^{*}\right)^{r}\right\}=(0,1,1) \\
& \tilde{x}^{*}=\left\{\tilde{x}_{2}^{*}=\left(x_{2}^{*}\right)^{p},\left(x_{2}^{*}\right)^{q},\left(x_{2}^{*}\right)^{r}\right\}=(0,0,0)
\end{aligned}
$$

The triangular fuzzy number ${\widetilde{Z_{1}}}^{*}=(0,0.66667,4)$

Now similarly the second part the problem $(5.19 \mathrm{~b})$ is as follows

$\tilde{x}^{*}=\left\{\check{x}_{1}^{*}=\left(x_{1}^{*}\right)^{p},\left(x_{1}^{*}\right)^{q},\left(x_{1}^{*}\right)^{r}\right\}=(0,1,1)$

$\tilde{x}^{*}=\left\{\tilde{x}_{2}^{*}=\left(x_{2}^{*}\right)^{p},\left(x_{2}^{*}\right)^{q},\left(x_{2}^{*}\right)^{r}\right\}=(0,0,0)$

The triangular fuzzy

number ${\widetilde{Z_{2}}}^{*}=(0,0.66667,4)$. The triangular fu zzy number $\widetilde{Z}^{*}=(0,0.44448889,16)$ The obtained result is exactly the optimal value of the problem which start with the original problem.

\section{Conclusions}

In the past few years, a growing interest has been shown in Fuzzy Quadratic fractional programming problem and currently there are several methods for solving FFQFP problems with non-negativity restrictions. By using proposed method, the FFQFP problem may be transformed into its equivalent three crisp linear fractional programming problems. To show the efficiency of our proposed method a practical problem has been illustrated.

\section{References}

[1] Abdulrahim B. K., (2017) "Using Feasible Directions To Solve Quadratic Fractional Programming Problems", An Academic and scientific journal issued by Garmyan university, Journal of Garmian university, Vol.1, No.12, PP.56-71, ISSN 2310-0087.

[2] Das S. K. and Mandal T., (2017) "A MOLFP Method for Solving Linear Fractional Programming under Fuzzy Environment", International Journal of Research in Industrial Engineering, Int. J. Res. Ind. Eng., Vol. 6, No. 3, PP. 202- 322.

[3] Dutta, D., Tiwari, R. N., \& Rao, J. R. (1992), "Multiple objective linear fract ional programming a fuzzy set theoretic approach", Fuzzy sets and Systems,Vo. 52, No. 1, PP.39-45.

[4] Fukushima M., and Hayashi Sh., (2008) "Quadratic Fractional Programming Problems with Quadratic Constraints", Department of Applied Mathematics and Physics, Graduate School of Informatics, Kyoto University.

[5] Lachhwani K., (2014) "FGP Approach to Multi Objective Quadratic Fractional Programming Problem", Int. J. Industrial Mathematics (ISSN 2008-5621), Article ID IJIM-00438, Vol. 6, No. 1, PP.4957. 
[6] Osman M. S., Emam O. E. and El Sayed M. A., (2017) "Multi-level Multi-objective Quadratic Fractional Programming Problem with Fuzzy Parameters: A FGP Approach", Asian Research Journal of Mathematics, Article no. ARJOM.34864, ISSN: 2456-477X, Vo.5, No.3, PP.1-19.

[7] Rashidul Hasan M. and Babul Hasan M., (2014) "An Alternative Method for Solving Quadratic Fractional Programming Problems with Homogenous Constraints", Journal of Emerging Trends in Engineering and Applied Sciences (JETEAS), (ISSN: 2141-7016), Vo.5, No.1, PP. 11-19.

[8] Stancu-Minasian, I. M., and Pop, B. (2003) "On a fuzzy set approach to solving multiple objective linear fractional programming problem” Fuzzy sets and systems, Vol.134, No.3, PP.397-405.

[9] Veeramani, C., and Sumathi, M. (2014) "Fuzzy mathematical programming approach for solving fuzzy linear fractional programming problem”, RAIRO-Operations research, Vo.48, No.1, PP.109-122. 